 \\ Human, Earth, and Future
}

ISSN: 2785-2997

Vol. 2, No. 2, June, 2021

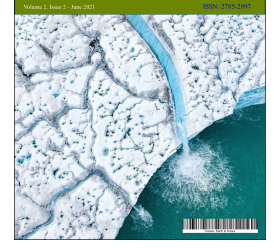

\section{Analytical Development of an Improved Inflow Performance Relationship (IPR) Model for Solution Gas Drive Reservoirs}

\author{
Temitayo Sheriff Adeyemi ${ }^{1 *} \mathbb{D}$, Deborah Oluwatosin Rufus ${ }^{1}$ \\ ${ }^{1}$ Department of Petroleum Engineering, University of Ibadan, Ibadan, Nigeria
}

Received 11 March 2021; Revised 17 May 2021; Accepted 25 May 2021; Published 01 June 2021

\begin{abstract}
Attempts have been made by many authors to develop an inflow performance relationship model suitable for solution gas drive reservoirs. However, they have not been as successful as most of the developed models suffer from certain degrees of inaccuracies, and this necessitates the need for an improved model as the economic analysis of an oilfield greatly depends on the ability to accurately forecast future production. Therefore, the objective of this research is to develop an improved inflow performance relationship model for solution gas reservoirs by employing a purely analytical approach and to compare the performance of the developed model with that of the existing IPR models (Vogel, Wiggins, Fetkovich, and Klins and Majher). A series expansion of the pseudo-steady state solution of the equation that governs fluid flow in reservoirs of radial geometry is obtained using Taylor's method, and the infinite series obtained is truncated after a reasonable number of terms to ensure a high degree of accuracy while also avoiding computational complexity. Moreover, the unknown coefficients in the truncated series were determined using the available reservoir fluid data. Finally, statistical analysis was carried out to determine the degree of deviation of the new and existing IPR models from the actual IPR. This analysis shows that the improved model (with an average coefficient of determination of 0.97) outperforms the existing IPR models to which it was compared. Therefore, the improved model is recommended for situations where extreme accuracy is of the utmost importance.
\end{abstract}

Keywords: Inflow Performance Relationship; Solution Gas Drive Reservoirs; Pseudosteady-state Flow.

\section{Introduction}

The Inflow Performance Relationship, or IPR, is defined as the working relationship between the production rate, reservoir pressure, and the bottom-hole flowing pressure. In 1954, Gilbert first proposed a well analysis using this relationship. IPR is operational in the pressure range between the average reservoir pressure and atmospheric pressure. The flow rate corresponding to the atmospheric pressure is defined as the absolute open flow potential of the well, whereas the flow rate at the average reservoir pressure bottom-hole is always zero [1].

Inflow Performance Relationships (IPRs) are pressure-rate relationships that are used to predict the performance of oil and gas wells. Vogel was one of the first people to propose an IPR for predicting the performance of oil wells [2]. This IPR was instantaneously accepted in the industry because it is easy to apply and yields reasonable results. His work is centred around Weller's approximations, which assume that the tank-oil de-saturation rate is the same at every point in the reservoir at any given time. The Vogel relationship assumes the following equation:

\footnotetext{
* Corresponding author: spegenius@gmail.com

$>$ This is an open access article under the CC-BY license (https://creativecommons.org/licenses/by/4.0/).

(C) Authors retain all copyrights.
} 
$\frac{q_{0}}{q_{0 \max }}=1-0.2\left(\frac{P_{w f}}{P_{R}}\right)-0.8\left(\frac{P_{w f}}{P_{R}}\right)^{2}$

where $q_{0}$ is the producing rate of oil corresponding to a particular flowing bottom-hole pressure $P_{w f}$. ' $P_{R}$ ' is an unchanged reservoir pressure and $q_{0}$, max is the production rate when the flowing bottom-hole pressure is equal to zero.

For wells whose pressure is below bubble point pressure, the following mathematical relationship was used by Fetkovich [3]:

$q_{0}=C\left(P_{R}^{2}-P_{w f}^{2}\right)^{n}$

where $P_{R}$ is the reservoir pressure at the time of discovery (before production starts), C and $\mathrm{n}$ are constants determined by empirical techniques.

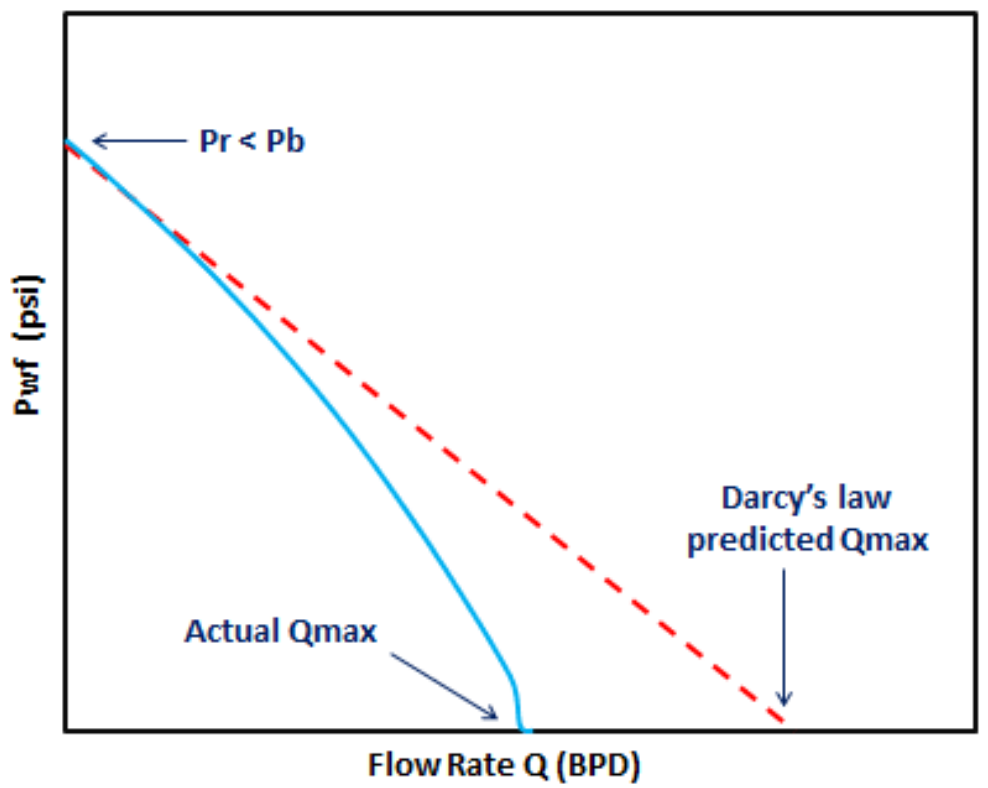

Figure 1. Comparing the straight line IPR predicted by Darcy's law with the observed actual relationship

Furthermore, other authors such as Klins \& Majcher (1992), Wiggins (1992), and Sukarno \& Tobing (1995) [4-6] also attempted to develop an IPR model for solution gas drive reservoirs by employing empirical method [7, 8]. In addition, Wiggins et al. (1996), and Archer et al. (2003) [9, 10] carried out research works to improve the Vogel's IPR model by employing a semi-analytical approach. However, the focus of this research is on how a purely analytical approach can be employed to develop an IPR model that outperforms other existing models [11-14].

\section{Research Methodology}

The flow chart below shows the processes involved in developing the improved inflow performance relationship model presented in this paper.
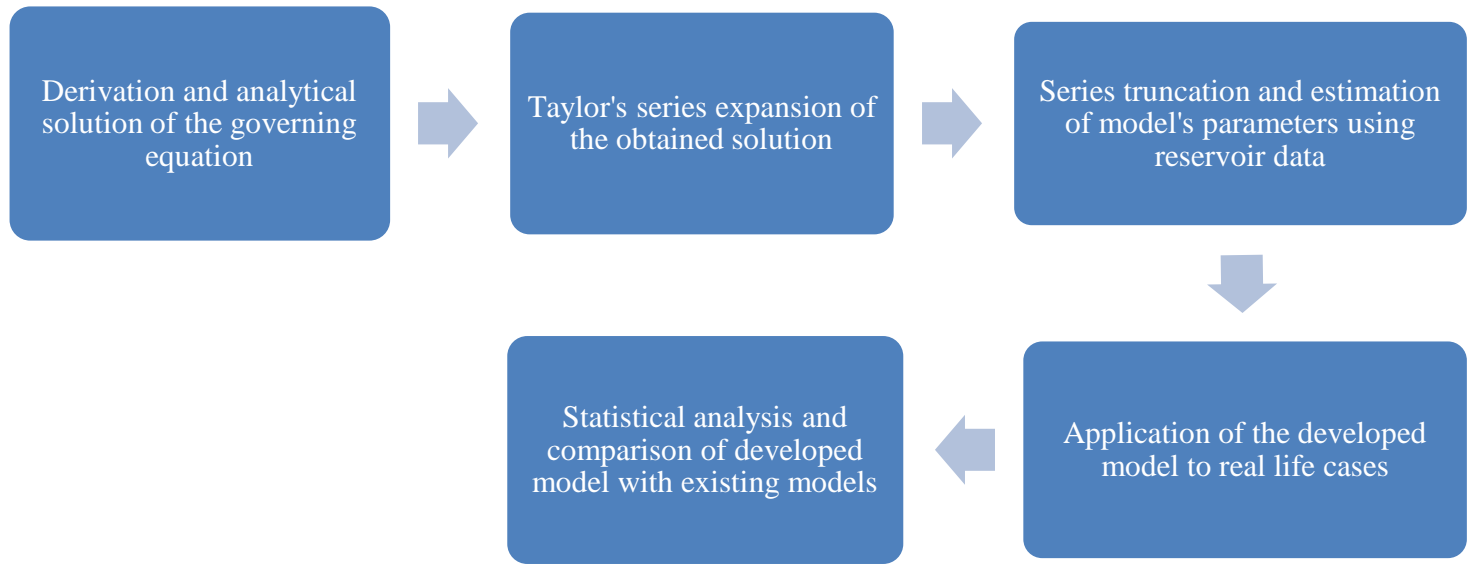

Figure 2. Flowchart of the research methodology 


\subsection{Theoretical Development}

If we consider a flow element of thickness, 'dr' and positioned at a radius ' $r$ ' from the centre of the well. Applying the principle of conservation of mass to the porous element, we have the following expressions;

$\left[\begin{array}{c}\text { Mass entering } \\ \text { Volume element } \\ \text { during interval }(\Delta t)\end{array}\right]-\left[\begin{array}{c}\text { Mass leaving } \\ \text { Volume element } \\ \text { during interval }(\Delta t)\end{array}\right]=\left[\begin{array}{c}\text { rate of mass } \\ \text { accumulation } \\ \text { during interval }(\Delta t)\end{array}\right]$

The mass influx into the porous element during a time interval $\Delta t$ is expressed as follows:

$(\operatorname{mass})_{m}=\Delta t(A V \rho)_{r+d r}$

where; $V=$ velocity of flowing fluid, $\rho=$ Fluid density, $A=$ Are, $t=$ Time.

The inlet area of the element can be expressed as;

$A r+d r=2 \pi \Delta t(r+d r) h(v \rho)_{r+d r}$

The mass flowing out of the porous element is given by;

$(\text { Mass })_{\text {out }}=2 \pi \Delta t r h(v \rho)_{r}$

The volume $\mathrm{V}$ of the porous element is expressed as;

$V=\pi r^{2} h$

$\frac{d v}{d r}=2 \pi h \quad$ or

$d v=(2 \pi r h) d r$

Total mass accumulation during $\Delta t$ is $d V\left[(\theta \rho)_{t+\Delta t}-(\theta \rho)_{t}\right]$

Substituting an expression for $\mathrm{dv}$, we have the total mass accumulation as;

$(2 \pi r h) d r\left[(\theta \rho)_{t+\Delta t}-(\theta \rho)_{t}\right]$

Further substitutions yield the following expression;

$2 \pi h(r+d r) \Delta t(\theta \rho)_{r+d r}-2 \pi h r \Delta t(\theta \rho)_{r}=(2 \pi r h) d r\left((C \theta \rho)_{t+\Delta t}-(\theta \rho)_{t}\right)$

Dividing Equation 9 by $(2 \pi r h) d r$ and simplifying gives:

$\frac{1}{(r) d r}\left[C r+d r(v \rho)_{r+d r}-r(v \rho)_{r}\right]=\frac{1}{\Delta t}\left[(\theta \rho)_{t+\Delta t}-(\theta \rho)_{t}\right]$

or;

$\frac{1}{r} \frac{\partial}{\partial r}[r(v \rho)]=\frac{\partial}{\partial t}(\theta \rho)$

The Equation 11 above is known as continuity equation and expresses the conservation of mass principle in radial geometry. The transport equation, which is the Darcy's law is expressed as follows;

$V=(0.001127) \frac{K}{\mu} \frac{\partial p}{\partial r}$

or;

$V=(0.006328) \frac{K}{\mu} \frac{\partial p}{\partial r}$

Combining Equations 11 and 13 results in; 
$\frac{0.006328}{r} \frac{\partial}{\partial r}\left[\frac{K}{\mu}(\rho r) \frac{\partial p}{\partial r}\right]=\frac{\partial}{\partial r}(\theta \rho)$

By expanding the RHS we have;

$\frac{\partial}{\partial t}(\theta \rho)=\theta \frac{\partial \rho}{\partial t}+\rho \frac{\partial \theta}{\partial t}$

The relationship between porosity and formation compressibility is given by the equation below;

$C f=\frac{1}{\theta} \frac{\partial \theta}{\partial p}$

$\frac{\partial \theta}{\partial t}=\frac{\partial \theta}{\partial p} \frac{\partial p}{\partial t}$

Substituting Equation 16 into this equation, we have the equation below;

$\frac{\partial \theta}{\partial t}=\theta C f \frac{\partial p}{\partial t}$

Finally, further substitutions and simplifications gives:

$\left[\frac{0.006328}{r}\right] \frac{\partial}{\partial r}\left(\frac{K}{\mu}(r \rho) \frac{\partial p}{\partial r}\right)=\rho \theta C f \frac{\partial p}{\partial t}+\theta\left(\frac{\partial p}{\partial t}\right)$

Equation 19 is the partial differential equation (PDE) that governs laminar flow of incompressible fluids in porous media. Modifications are required for compressible and slightly compressible fluids.

$\left[\frac{0.006328}{r} K\right] \frac{\partial}{\partial r}\left(r \rho \frac{\partial p}{\partial r}\right)=\rho \theta C f \frac{\partial p}{\partial t}+\theta\left(\frac{\partial \rho}{\partial t}\right)$

$\frac{0.006328}{r}\left(\frac{K}{\mu}\right)\left[\frac{\rho}{r} \frac{\partial P}{\partial r}+\rho \frac{\partial^{2} P}{\partial r^{2}}+\frac{\partial P}{\partial r}\left(\frac{\partial \rho}{\partial r}\right)\right]=\rho \theta C f\left[\frac{\partial P}{\partial t}\right]+\theta\left(\frac{\partial \rho}{\partial t}\right)$

Further simplification gives;

$\frac{0.006328}{r}\left(\frac{K}{\mu}\right)\left[\frac{\rho}{r} \frac{\partial P}{\partial r}+\rho \frac{\partial^{2} P}{\partial r^{2}}+\left(\frac{\partial P}{\partial r}\right)^{2}\left(\frac{1}{\rho} \frac{\partial \rho}{\partial P}\right)\right]=\rho \theta C f\left[\frac{\partial P}{\partial t}\right]+\theta\left(\frac{\partial P}{\partial t}\right)\left(\frac{\partial \rho}{\partial P}\right)$

By dividing through by the fluid's density, we have;

$\frac{0.006328}{r}\left(\frac{K}{\mu}\right)\left[\frac{1}{r} \frac{\partial P}{\partial r}+\frac{\partial^{2} P}{\partial r^{2}}+\left(\frac{\partial P}{\partial r}\right)^{2}\left(\frac{1}{\rho} \frac{\partial \rho}{\partial P}\right)\right]=\theta C f\left[\frac{\partial P}{\partial t}\right]+\theta\left(\frac{\partial P}{\partial t}\right)\left(\frac{1}{\rho} \frac{\partial \rho}{\partial P}\right)$

$C=\frac{1}{\rho} \frac{\partial \rho}{\partial P}$

By combining Equations 22 and 23, we have;

$0.006328\left(\frac{K}{\mu}\right)\left[\frac{\partial^{2} P}{\partial r^{2}}+\frac{1}{r} \frac{\partial P}{\partial r}+C\left(\frac{\partial P}{\partial r}\right)^{2}\right]=\theta C f\left[\frac{\partial P}{\partial t}\right]+\theta C\left(\frac{\partial P}{\partial t}\right)$

The term $C\left[\frac{\partial P}{\partial r}\right]^{2}$ is regarded very small and is ignored:

$0.006328\left(\frac{K}{\mu}\right)\left[\frac{\partial^{2} P}{\partial r^{2}}+\frac{1}{r} \frac{\partial P}{\partial r}\right]=\theta(C f+C)\left[\frac{\partial P}{\partial t}\right]$

Total compressibility, $\mathrm{C}_{\mathrm{t}}$, is defined as: 
$C t=C+C f$

Finally, combining Equations $19 \mathrm{~b}$ and 25 gives;

$\frac{\partial^{2} P}{\partial r^{2}}+\frac{1}{r} \frac{\partial P}{\partial r}=\frac{\theta \mu C f}{0.006328 K} \frac{\partial P}{\partial t}$

Equation 27 is known as the diffusivity equation. When time is changed from days to hours, it takes the following form;

$\frac{\partial^{2} P}{\partial r^{2}}+\frac{1}{r} \frac{\partial P}{\partial r}=\frac{\theta \mu C t}{0.000264 K} \frac{\partial P}{\partial t}$

$\eta=\frac{0.000264 K}{\theta \mu C t}$

By making the above substitution, Equation 28 can be written more conveniently as follows:

$\frac{\partial^{2} P}{\partial r^{2}}+\frac{1}{r} \frac{\partial P}{\partial r}=\frac{1}{\eta} \frac{\partial P}{\partial t}$

For Pseudo-steady state flow, pressure changes with time at a constant rate;

$\left(\frac{\partial P}{\partial t}\right)=$ constant

$\frac{\partial P}{\partial t}=\frac{-0.23396 q}{C_{t} \pi r_{e}^{2} h \theta}$

$\frac{\partial^{2} P}{\partial r^{2}}+\frac{1}{r} \frac{\partial P}{\partial r}=\frac{\theta \mu C f}{0.006328 K} \frac{0.23396 q}{C_{t} A h \theta}$

OR

$\frac{\partial^{2} P}{\partial r^{2}}+\frac{1}{r} \frac{\partial P}{\partial r}=\frac{-887.22 q \mu}{A h K}$

Equation $32 \mathrm{~b}$ can be expressed as:

$\frac{1}{r} \frac{\partial}{\partial r}\left(\frac{r \partial P}{\partial r}\right)=\frac{-887.22 q \mu}{\left(\pi r_{e}\right) h k}$

Integrating equation 33 gives:

$\frac{r \partial P}{\partial r}=\frac{-887.22 q \mu}{\left(\pi r_{e}^{2}\right) h k} \frac{r^{2}}{2}+C_{1}$

where $C_{1}$, the constant of the integration can be evaluated by imposing the outer no-flow boundary condition (i.e. $\left.(\partial P / \partial r)_{r_{e}}=0\right)$ on the above relation to give:

$C_{1}=\frac{141.2 q \mu}{\pi h k}$

Combining Equations 34 and 35 gives:

$\partial P / \partial r=\frac{141.2 q \mu}{h k}\left(\frac{1}{r}-\frac{1}{r_{e}^{2}}\right)$

Integrating once again: 
$\int_{P_{w f}}^{P_{1}} d p=\frac{141.2 q \mu}{h k} \int_{r_{w}}^{r_{e}}\left(\frac{1}{r}-\frac{1}{r_{e}^{2}}\right) d r$

By integrating the above expression and by assuming that $\left(\frac{r_{w}^{2}}{r_{e}^{2}}\right)$ is negligible, we have;

$\left(P_{1}-P_{w f}\right)=\frac{141.2 q \mu}{h k}\left(\ln \left(\frac{r_{e}}{r_{w}}\right)-\frac{1}{2}\right)$

Solving for rate in the above equation from gives:

$Q=\frac{0.00708 k h\left(P_{r}-P_{w f}\right)}{\mu \beta\left(\ln \left(\frac{r_{e}}{r_{w}}\right)-0.5\right)}$

The volumetric average reservoir pressure $\mathrm{P}_{1}$ is usually used in calculating the liquid flow rate under the Pseudosteady state flowing condition. Introducing $\mathrm{P}$, into Equation 39 gives;

$Q=\frac{0.00708 k h\left(P_{r}-P_{w f}\right)}{\mu \beta\left(\ln \left(\frac{r_{e}}{r_{w}}\right)-0.75\right)}$

Introducing skin effect into the equation, the equation yields:

$Q=\frac{0.00708 k h\left(P_{r}-P_{w f}\right)}{\mu_{0} \beta_{0}\left(\ln \left(\frac{r_{e}}{r_{w}}\right)-0.75+s\right)}$

$\Delta P=P_{r}-P$

$\Delta P=f(P)$

$\frac{d(\Delta P)}{P}=0-1$

$\frac{d(\Delta P)}{P}=-1$

$d(\Delta P)=-d P$

$K_{0}=K K_{r_{0}}$

Taking $-\frac{0.00708 k h\left(P_{r}-P_{w f}\right)}{\left(\ln \left(\frac{r_{e}}{r_{w}}\right)-3 / 4+s\right)}$ as C;

$q=C \int_{P_{1}}^{P_{2}}\left(\frac{K_{r_{0}}}{\mu_{0} \beta_{0}}\right) d p$

$q=C \int_{0}^{P_{r}-P} \frac{K_{r_{0}}}{\mu_{0} \beta_{0}} d p$

$q=C \int_{0}^{\Delta P} \frac{K_{r_{0}}}{\mu_{0} \beta_{0}} d p$

$d p=-d(\Delta P)$

$q=C \int_{0}^{\Delta P} \frac{K_{r_{0}}}{\mu_{0} \beta_{0}} d(\Delta P)$

Normalizing the equations by dividing through by $P_{r}$; 
$q=C P_{r} \int_{0}^{\frac{\Delta P}{P_{r}}} \frac{K_{r_{0}}}{\mu_{0} \beta_{0}} d\left(\frac{\Delta P}{P_{r}}\right)$

$\frac{\Delta P}{P_{r}}=P_{D}($ dimensionless pressure $)$

$q=C P_{r} \int_{0}^{P_{D}} \frac{K_{r_{0}}}{\mu_{0} \beta_{0}} d\left(P_{D}\right)$

Recall that $\int_{a}^{b} f(x)=f(b)-f(a) ; f(a)-f(a)=0$

$q=d\left(P_{D}\right)$

$\frac{d q\left(P_{D}\right)}{d P_{D}}=\left[\frac{C P_{r} K_{r_{0}}}{\mu_{0} \beta_{0}}\right]^{1}$

$q^{1}\left(P_{D}\right)=\left[\frac{C P_{r} K_{r_{0}}}{\mu_{0} \beta_{0}}\right]$

Expanding using Taylor's series, the differential becomes:

$q\left(P_{D}\right)=q\left(P_{D}\right)+\left(P_{D}-P_{D_{0}}\right) q^{I}\left(P_{D_{0}}\right)+\frac{\left(P_{D}-P_{D_{0}}\right)^{2}}{2 !} q^{I I}\left(P_{D_{0}}\right)+\cdots$
$q\left(P_{D}\right)=P_{D} q^{I}\left(P_{D_{0}}\right)+\frac{P_{D}{ }^{2}}{2 !} q^{I I}\left(P_{D_{0}}\right)+\frac{P_{D}{ }^{3}}{3 !} q^{I I I}\left(P_{D_{0}}\right)+\cdots$
$q\left(P_{D}\right)=P_{D}\left(\frac{C P_{r K_{r_{0}}}}{\mu_{0} \beta_{0}}\right)^{1}+\frac{P_{D}{ }^{2}}{2 !}\left(\frac{C P_{r K_{r_{0}}}}{\mu_{0} \beta_{0}}\right)^{1}+\frac{P_{D}{ }^{3}}{3 !}\left(\frac{C P_{r K_{r_{0}}}}{\mu_{0} \beta_{0}}\right)^{I I}+\frac{P_{D}{ }^{4}}{4 !}\left(\frac{C P_{r K_{r_{0}}}}{\mu_{0} \beta_{0}}\right)^{I I I}+\frac{P_{D}{ }^{5}}{5 !}\left(\frac{C P_{r K_{r_{0}}}}{\mu_{0} \beta_{0}}\right)^{I V}+\Sigma$

where $\Sigma$ is the truncation term;

$q\left(P_{D}\right)=q_{0}$

$P_{D}=\frac{P_{r}-P_{w f}}{P_{r}}$

At $q_{0 \max }, P_{w f}=0, P_{D}=1$.

$q_{0 \max }=\frac{C P_{r K_{r_{0}}}}{\mu_{0} \beta_{0}}+\frac{1}{2 !}\left(\frac{C P_{r K_{r_{0}}}}{\mu_{0} \beta_{0}}\right)^{1}+\frac{1}{3 !}\left(\frac{C P_{r K_{r_{0}}}}{\mu_{0} \beta_{0}}\right)^{I I}+\frac{1}{4 !}\left(\frac{C P_{r K_{r_{0}}}}{\mu_{0} \beta_{0}}\right)^{I I I}+\frac{1}{5 !}\left(\frac{C P_{r K_{r_{0}}}}{\mu_{0} \beta_{0}}\right)^{I V}+\Sigma$

$q_{0} / q_{0} \max =C_{1} P_{D}+C_{2} P_{D}^{2}+C_{3} P_{D}^{3}+C_{4} P_{D}^{4}+C_{5} P_{D}^{5}$

Let Equation 60 be equal to $\mathrm{m}$.

$C_{1}=\frac{\frac{C P_{r K_{r_{0}}}}{\mu_{0} \beta_{0}}}{m}$

$C_{2}=\frac{\frac{C P_{r K_{r_{0}}}}{\mu_{0} \beta_{0}}+\frac{1}{2 !}\left(\frac{C P_{r K_{r_{0}}}}{\mu_{0} \beta_{0}}\right)}{m}$

$C_{3}=\frac{\frac{C P_{r K_{r_{0}}}}{\mu_{0} \beta_{0}}+\frac{1}{2 !}\left(\frac{C P_{r K_{r_{0}}}}{\mu_{0} \beta_{0}}\right)^{1}+\frac{1}{3 !}\left(\frac{C P_{r K_{r_{0}}}}{\mu_{0} \beta_{0}}\right)^{I I}}{m}$ 
$C_{4}=\frac{\frac{C P_{r K_{r_{0}}}}{\mu_{0} \beta_{0}}+\frac{1}{2 !}\left(\frac{C P_{r K_{r_{0}}}}{\mu_{0} \beta_{0}}\right)^{1}+\frac{1}{3 !}\left(\frac{C P_{r K_{r_{0}}}}{\mu_{0} \beta_{0}}\right)^{I I}+\frac{1}{4 !}\left(\frac{C P_{r K_{r_{0}}}}{\mu_{0} \beta_{0}}\right)^{I I I}}{m}$

$C_{5}=\frac{\frac{C P_{r K_{r_{0}}}}{\mu_{0} \beta_{0}}+\frac{1}{2 !}\left(\frac{C P_{r K_{r_{0}}}}{\mu_{0} \beta_{0}}\right)^{1}+\frac{1}{3 !}\left(\frac{C P_{r K_{r_{0}}}}{\mu_{0} \beta_{0}}\right)^{I I}+\frac{1}{4 !}\left(\frac{C P_{r K_{r_{0}}}}{\mu_{0} \beta_{0}}\right)^{I I I}+\frac{1}{5 !}\left(\frac{C P_{r K_{r_{0}}}}{\mu_{0} \beta_{0}}\right)^{I V}}{m}$

Solving for each coefficient, then we have;

$\frac{Q_{0}}{Q_{0} \max }=1-0.5372\left(\frac{P_{w f}}{P_{r}}\right)+0.4882\left(\frac{P_{w f}}{P_{r}}\right)^{2}-1.1037\left(\frac{P_{w f}}{P_{r}}\right)^{3}+0.3402\left(\frac{P_{w f}}{P_{r}}\right)^{4}-0.1857\left(\frac{P_{w f}}{P_{r}}\right)^{5}$

The Equation 61 is the improved IPR model.

\section{Results and Discussion}

The Equation 61 is an improved inflow performance relationship model for solution gas drive reservoirs. It was observed that the accuracy of the model increases with increase in the number terms in the series expansion. However, the increase in accuracy is not so significant after the sixth term. Therefore, the series was truncated after the sixth term to avoid unnecessary computations.

\subsection{Comparison with Existing Models}

The improved model's accuracy is compared with that of existing ones (Vogel', Fetkovich', Wiggins' and Klins and Majher).

Following the use of three case studies for validating the accuracy of the improved model, the actual data and the results obtained using the improved model, Vogel's, Wiggin's and Fetkovich's were plotted and the coefficient of determination was employed to estimate the degree of deviation of each model from actual field result. Three different reservoirs were considered and some of the results obtained are shown in the plots below:

\section{CASE 1}

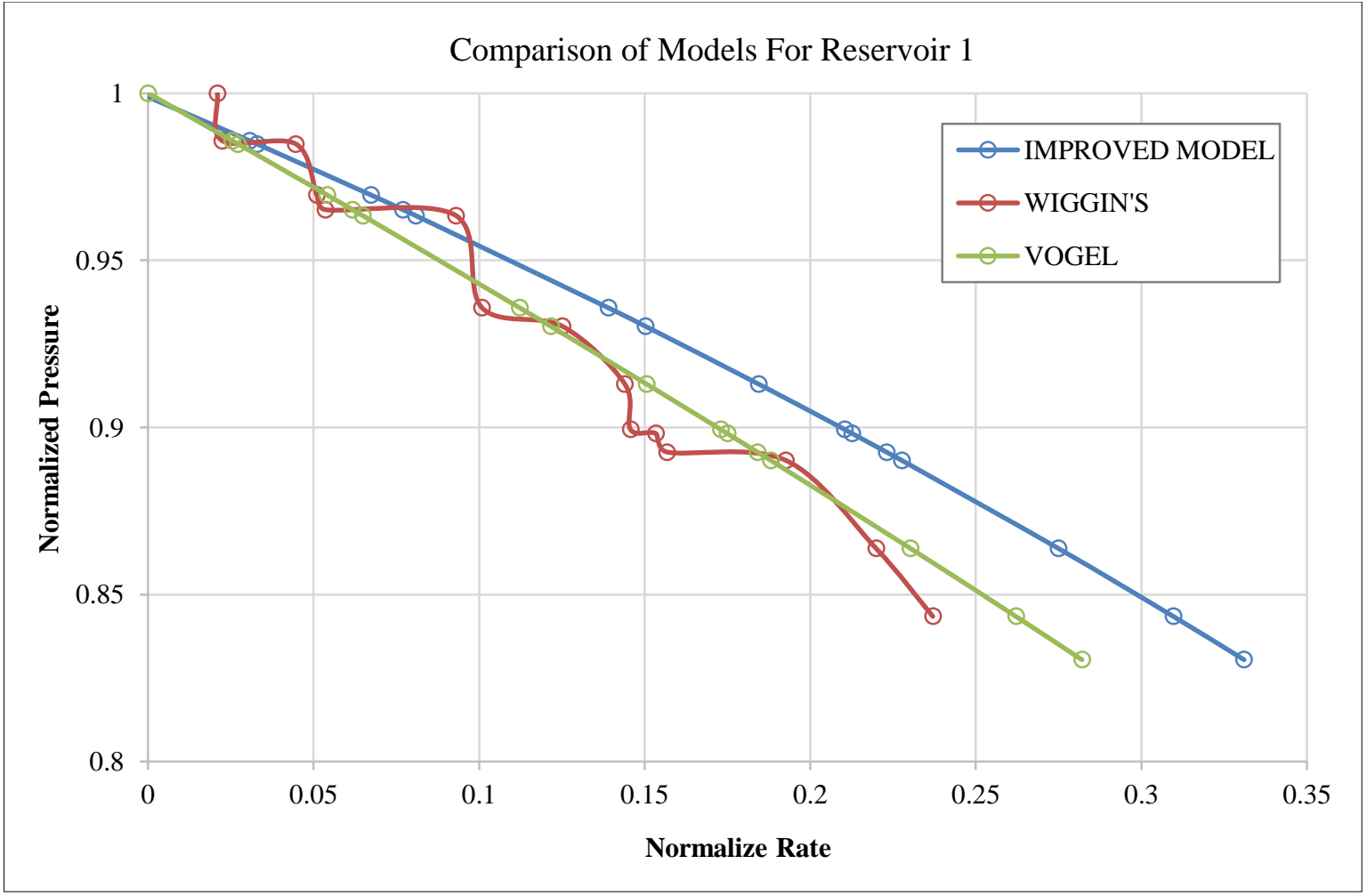

Figure 3. Plot of Normalized Pressure against Normalized Rate for Reservoir 1 


\section{CASE 2}

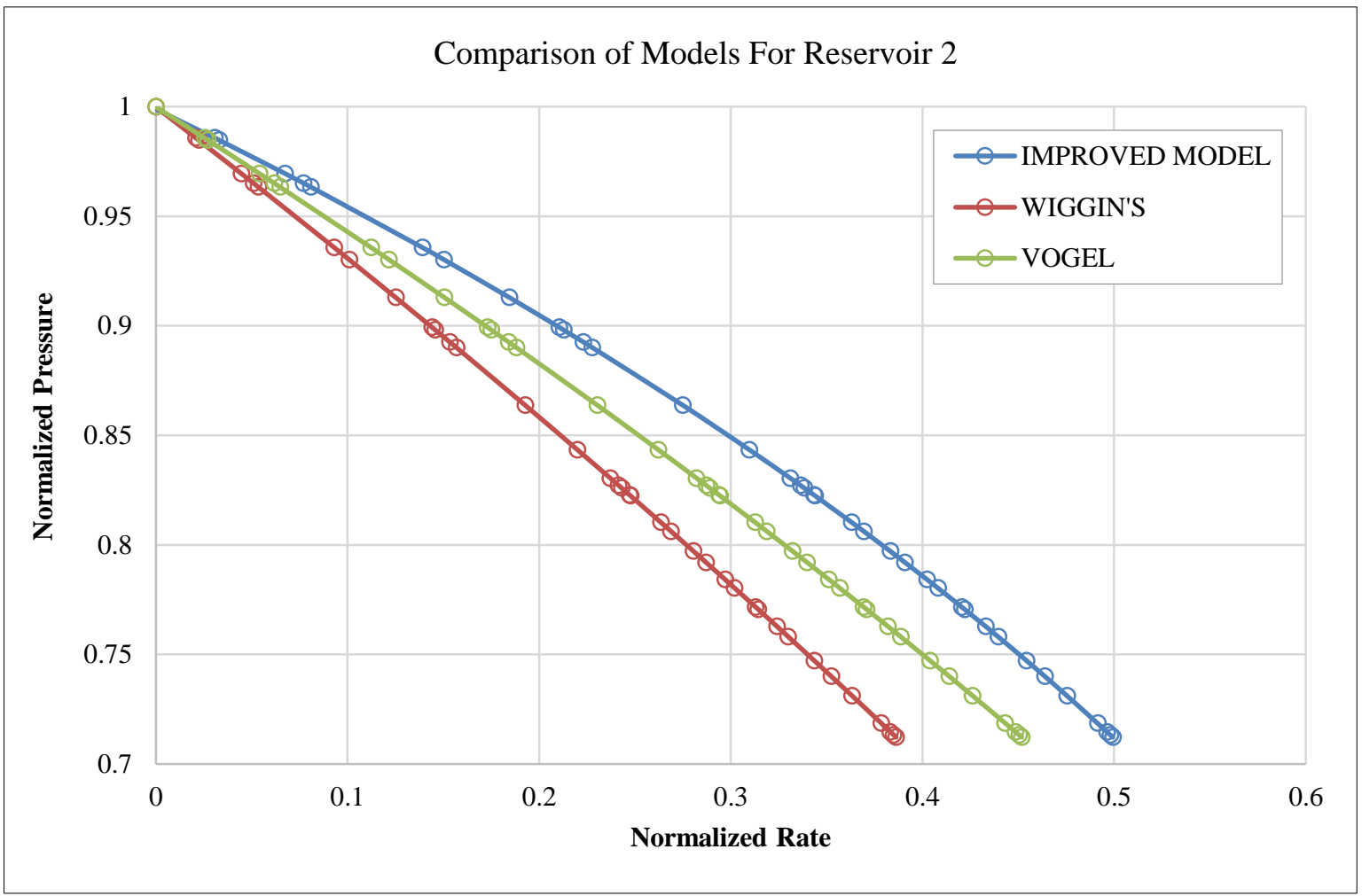

Figure 4. Plot of Normalized Pressure against Normalized Rate for Reservoir 2

\section{CASE 3}

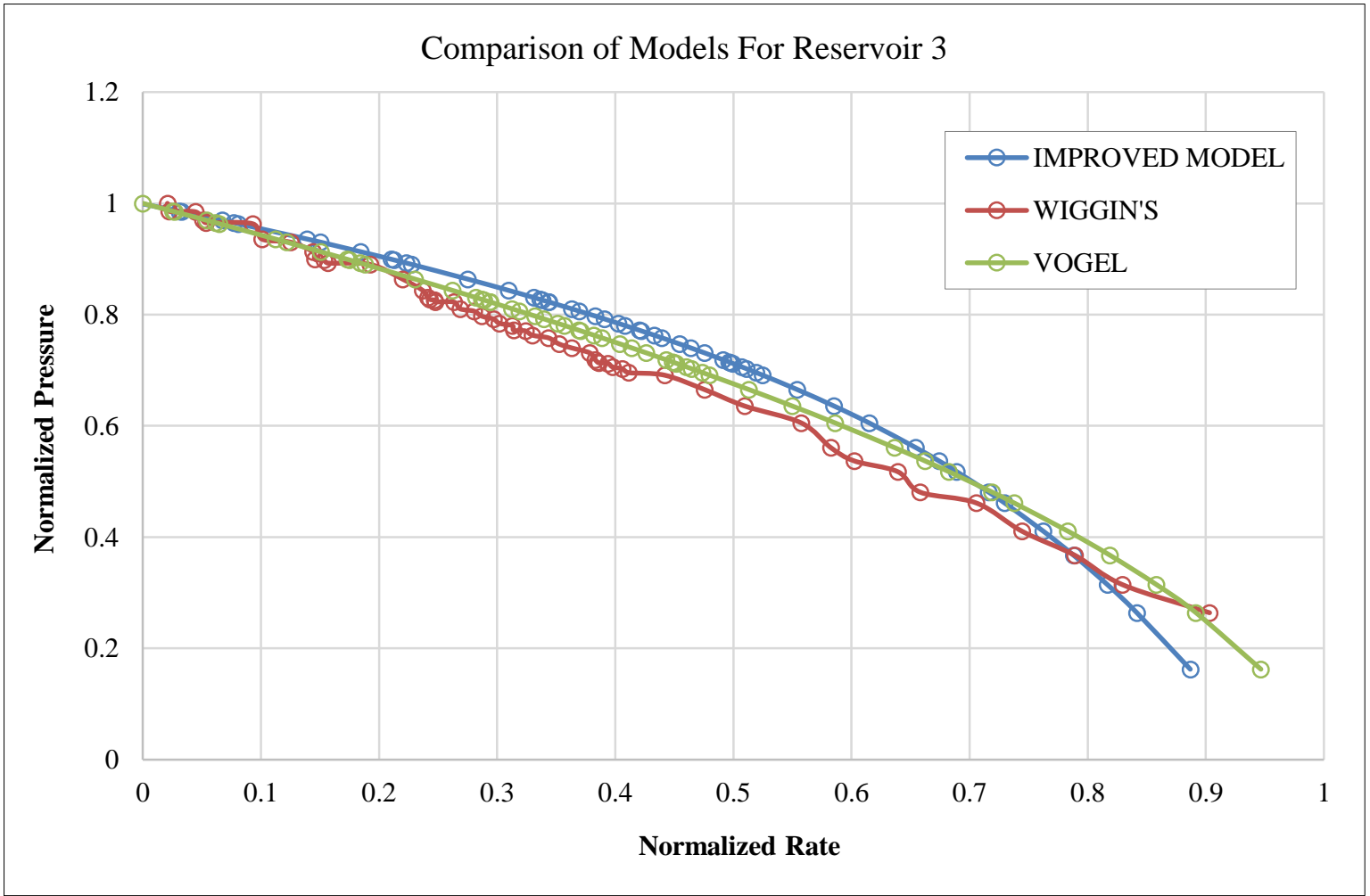

Figure 5. Plot of Normalized Pressure against Normalized Rate for Reservoir 3

\subsection{Statistical Analysis}

The following tables show the results of the statistical analysis of the results obtained for each of the reservoirs using the new and existing models. 
Table 1. Statistical analysis of results obtained for reservoir 1

\begin{tabular}{ccccc}
\hline Error analysis & NEW MODEL & VOGEL & FETKOVICH & WIGGINS \\
\hline $\mathrm{R}^{2}$ value & 0.902 & 0.446 & 0.815 & 0.333 \\
\hline
\end{tabular}

CASE 2

Table 2. Statistical analysis of results obtained for reservoir 2

\begin{tabular}{ccccc}
\hline Error analysis & NEW MODEL & VOGEL & FETKOVICH & WIGGINS \\
\hline $\mathrm{R}^{2}$ value & 0.950 & 0.928 & 0.915 & 0.908 \\
\hline
\end{tabular}

$\underline{C A S E} 3$

Table 3. Statistical analysis of results obtained for reservoir 3

\begin{tabular}{ccccc}
\hline Error analysis & NEW MODEL & VOGEL & FETKOVICH & WIGGINS \\
\hline $\mathrm{R}^{2}$ value & 0.972 & 0.967 & 0.958 & 0.911 \\
\hline
\end{tabular}

\section{Conclusion}

An improved inflow performance relationship model for solution gas drive reservoirs has been developed. The plots obtained when this model was applied to three different reservoir cases show a high degree of similarity to the ones obtained using the two widely accepted IPR models in the oil and gas industry. In addition, an approximately linear relationship was observed for the first two cases, while the third case shows a high degree of deviation from linearity. From these, we can conclude that for the first two cases, the reservoirs were still being produced above the bubble point pressure, while the third case is a typical scenario for a reservoir whose pressure had dropped below the bubble point pressure. Since the model performed excellently on both saturated and under-saturated reservoirs, we can conclude that the model can be used with a reasonable level of confidence to evaluate the performance of a solution gas drive reservoir at any stage of its production life. Furthermore, the statistical analysis of the results obtained using this model shows that it outperforms the existing inflow performance relationship models to which it was compared. Although more computationally intensive, this improved IPR model is recommended for solution gas reservoirs where accuracy is of paramount importance.

\section{Nomenclature}

$\begin{array}{llll}Q_{o} & \text { Oil, flow rate, } \mathrm{STB} / \mathrm{day} & P_{e} & \text { External pressure, } \mathrm{psi} \\ P_{w f} & \text { Bottom-hole flowing pressure, psi } & \mathrm{N} & \text { Oil viscosity, } \mathrm{cp} \\ B_{o} & \text { Oil formation volume factor, bbl/STB } & \mathrm{h} & \text { Thickness, } \mathrm{ft} \\ r_{e} & \text { External or drainage radius, } \mathrm{ft} & r_{w} & \text { Wellbore radius, } \mathrm{ft} \\ p & \text { Average reservoir pressure, psia } & \theta & \text { Porosity, fraction } \\ \rho & \text { Density, } \mathrm{lb} / \mathrm{ft}^{3} & v & \text { Fluid velocity, ft/day } \\ \Delta \mathrm{t} & \text { Time interval, days or hrs. } & k & \text { Absolute permeability, md } \\ k_{r o} & \text { Relative permeability to oil } & J & \text { Productivity index } \\ s & \text { Skin factor } & \mathrm{r} & \text { Radius, ft } \\ \mathrm{p} & \text { Pressure, psia } & c_{t} & \text { Total compressibility, psi }\end{array}$

\section{Declarations}

\subsection{Author Contributions}

Conceptualization was carried out by T.S.A. and D.O.R.; the research methodology was developed by T.S.A.; Data curation and formal analysis were also done by T.S.A.; D.O.R. carried out the results validation, visualization, and also prepared the original draft. Finally, T.S.A. wrote the review and also carried out the editing. All authors have read and agreed to the published version of the manuscript.

\subsection{Data Availability Statement}

The data presented in this study are available in article.

\subsection{Funding}

The authors received no financial support for the research, authorship, and/or publication of this article. 


\subsection{Institutional Review Board Statement}

Not applicable.

\subsection{Informed Consent Statement}

Not applicable.

\subsection{Declaration of Competing Interest}

The authors declare that there is no conflict of interests regarding the publication of this manuscript. In addition, the ethical issues, including plagiarism, informed consent, misconduct, data fabrication and/or falsification, double publication and/or submission, and redundancies have been completely observed by the authors.

\section{References}

[1] Gilbert, W. E. (1954). Flowing and gas-lift well performance. Drilling and Production Practice, 126-157, OnePetro, Texas, United States.

[2] Vogel, J. V. (1968). Inflow Performance Relationships for Solution-Gas Drive Wells. Journal of Petroleum Technology, 20(01), 83-92. doi:10.2118/1476-pa.

[3] Fetkovich, M. J. (1973). The isochronal testing of oil wells. Society of Petroleum Engineers - Fall Meeting of the Society of Petroleum Engineers of AIME, FM 1973. doi:10.2118/4529-ms.

[4] Klins, M. A., \& Majcher, M. W. (1992). Inflow performance relationships for damaged or improved wells producing under solution-gas drive. JPT, Journal of Petroleum Technology, 44(12), 1357-1363. doi:10.2118/19852-PA.

[5] Wiggins, M. L. (1992). Analytically Development of Vogel-Type Inflow Performance Relationships. In SPE 23580 Presented at SPE (pp. 18-20).

[6] Sukarno, P., \& Tobing, E. L. (1995). Inflow Performance Relationship for Perforated Wells Producing From Solution Gas Drive Reservoir. All Days. doi:10.2118/29312-ms.

[7] Ahmed, T. (2018). Reservoir Engineering Handbook (fourth edit.). Gulf Professional Publishing, Texas, United States. doi:10.1016/C2016-0-04718-6.

[8] Shi, C., \& Horne, R. N. (2008). Improved recovery in gas-condensate reservoirs considering compositional variations. In SPE Annual Technical Conference and Exhibition. OnePetro. doi:10.2118/115786-ms.

[9] Wiggins, M. L., Russell, J. E., \& Jennings, J. W. (1996). Analytical development of Vogel-type inflow performance relationships. SPE Journal, 1(4), 355-362. doi:10.2118/23580-PA.

[10] Archer, R. A., Del Castillo, Y., \& Blasingame, T. A. (2003). New Perspectives on Vogel Type IPR Models for Gas Condensate and Solution-Gas Drive Systems. All Days. doi:10.2118/80907-ms.

[11] Lu, N., Hou, J., Liu, Y., Barrufet, M. A., Bai, Y., Ji, Y., ... Zhou, K. (2019). Revised inflow performance relationship for productivity prediction and energy evaluation based on stage characteristics of Class III methane hydrate deposits. Energy, 189, 116211. doi:10.1016/j.energy.2019.116211.

[12] Zhang, S., Liu, H., Wang, Y., Sun, K., \& Guo, Y. (2021). A Novel Mathematical Model Considering Real Gas PVT Behavior to Estimate Inflow Performance Relationship of Gas Well Production. Energies, 14(12), 3594. doi:10.3390/en14123594.

[13] Fattah, K. A., Elias, M., El-Banbi, H. A., \& El-Tayeb, E.-S. A. (2014). New Inflow Performance Relationship for solution-gas drive oil reservoirs. Journal of Petroleum Science and Engineering, 122, 280-289. doi:10.1016/j.petrol.2014.07.021.

[14] Okotie, S., \& Ikporo, B. (2018). Inflow Performance Relationship. Reservoir Engineering, 339-354. doi:10.1007/978-3-03002393-5_9. 